OPEN ACCESS

Edited by:

YiXu,

Texas A\&M Health Science Center, United States

Reviewed by: Yanfei Chen,

Zhejiang University, China Yousong Peng,

Hunan University, China

*Correspondence:

Junrong Liang

liangjunrong@icdc.cn Baogang Xie xiebaogang49@zjxu.edu.cn Shuilin Sun

sunshuilin2280@126.com

${ }^{+}$These authors have contributed equally to this work

Specialty section:

This article was submitted to Microbiome in Health and Disease, a section of the journal Frontiers in Cellular and Infection Microbiology

Received: 18 June 2020 Accepted: 26 February 2021 Published: 06 April 2021

Citation: Yao X, Yu H, Fan G, Xiang $H$, Long L, Xu H, Wu Z, Chen M, Xi W, Gao Z, Liu C, Gong W, Yang A, Sun K, Yu R, Liang J, Xie B and Sun $S$ (2021) Impact of the Gut Microbiome on the Progression

of Hepatitis B Virus Related Acute-on-Chronic Liver Failure. Front. Cell. Infect. Microbiol. 11:573923. doi: 10.3389/fcimb.2021.573923

\section{Impact of the Gut Microbiome on the Progression of Hepatitis B Virus Related Acute-on-Chronic Liver Failure}

\author{
Xuebing Yao ${ }^{1+}$, Haiping $\mathrm{Yu}^{1+}$, Guoyin Fan ${ }^{2+}$, Haihong Xiang ${ }^{1}$, Lin Long ${ }^{1}$, Huili Xu ${ }^{1}$, \\ Zhiguo Wu ${ }^{1}$, Mingfa Chen ${ }^{1}$, Wenna Xi ${ }^{1}$, Zhen Gao ${ }^{1}$, Cuiyun Liu ${ }^{1}$, Wenlan Gong ${ }^{1}$, \\ Aoyu Yang ${ }^{1}$, Ke Sun ${ }^{1}$, Rongyan Yu ${ }^{1}$, Junrong Liang ${ }^{3 *}$, Baogang Xie ${ }^{4 *}$ and Shuilin Sun ${ }^{1 *}$ \\ ${ }^{1}$ Department of Infectious Diseases, The Second Affiliated Hospital of Nanchang University, Nanchang, China, ${ }^{2}$ Department of \\ Infectious Diseases, Nanchang Centers for Disease Control and Prevention, Nanchang, China, ${ }^{3}$ State Key Laboratory for Infectious \\ Disease Prevention and Control, National Institute for Communicable Disease Control and Prevention, Chinese Center for Disease \\ Control and Prevention, Beijing, China, ${ }^{4}$ Department of Pharmaceutics, Medical College of Jiaxing University, Jiaxing, China
}

The relationship between the progression of hepatitis B virus-related acute-on-chronic liver failure (HBV-ACLF) and the gut microbiota is poorly understood, and an HBV-ACLFrelated microbiome has yet to be identified. In this study alterations in the fecal microbiome of 91 patients with HBV-ACLF (109 stool samples), including a cohort of nine patients at different stages of HBV-ACLF, were determined by high-throughput $16 \mathrm{~S}$ rDNA sequencing. The operational taxonomic units and Shannon indexes indicated that the diversity and abundance of the gut microbiome significantly decreased with the progression of HBV-ACLF ( $p<0.05$ ). The relative abundance of the Bacteroidetes phylum in the microbiome was significantly reduced, whereas the abundance of potentially pathogenic bacteria, such as Veilonella, Streptococcus, Enterococcus, and Klebsiella, was highly enriched in the HBV-ACLF group compared with the healthy control group. The abundance of Bacteroidetes was negatively correlated with the level of serum alpha fetoprotein, and the abundance of Veilonella was positively correlated with serum total bilirubin (TBIL). Furthermore, the abundance of Coprococcus was significantly negatively correlated with the level of serum TBIL and the international normalized ratio and positively correlated with prothrombin time activity. Our findings suggest that the gut microbiota plays an important role in the development of HBV-ACLF.

Keywords: fecal microbiome, hepatitis B virus, acute-on-chronic liver failure, biomedical indicators, metabolites

\section{INTRODUCTION}

Hepatitis B virus (HBV) infection is a major public health problem worldwide. The number of patients infected with HBV was estimated to be 93 million in China, and a small proportion of the chronic infections progress to severe hepatitis and liver failure known as HBV-associated acute-onchronic liver failure (HBV-ACLF) (Tsai et al., 2015). However, HBV-ACLF is a devastating disease with high mortality (Wlodzimirow et al., 2012; Bajaj et al., 2014a). With the progression of liver 
failure, the liver tissue suffers damages from immune injury, ischemic anoxia, and endotoxemia (Zang et al., 2000; Uesugi et al., 2002; Xing et al., 2007; Chen et al., 2021). The onset and progression of HBV-ACLF have four phases (Ye and Gao, 2009): pre-rise, ascending, plateau, and recovery. In the early stage of ACLF (pre-rise phase), the body suffers from immune damage, as well as ischemia and hypoxia injury. In the middle and late stages of the ascending phase, endotoxemia causes a "second attack" to the body. In the late stages of the plateau phase and early recovery phase, the immune system is suppressed, mainly suffering from the damage from endotoxemia. Understanding the HBV-ACLF related microbiome changes may open new avenue for treatment targeted at different phases of HBVACLF and better management of the highly variable clinical course of HBV-ACLF (Chen EQ et al., 2015).

HBV-ACLF patients have been shown to suffer intestinal flora imbalance, with an increase in intestinal permeability and high endotoxemia (Tilg et al., 2016). The outcomes of patients with HBV-ACLF can be significantly improved if interventions are timely and appropriately provided (Ott et al., 2017). Recently, many studies have demonstrated that HBV-ACLF development is closely associated with disorders in immune function, intestinal bacterial translocation (BT), gut dysbiosis and inflammation (Verbeke et al., 2011; Wang and Li, 2015; Preveden et al., 2017; Zhang et al., 2018). A previous study showed that stimulation by translocated bacteria or bacterial products (e.g., lipopolysaccharide, lipoteichoic acid, peptidoglycans, and bacterial DNA) can activate the innate immune system and modify the adaptive immune system, resulting in inflammation, liver cell apoptosis, and progression to liver failure (Sandler et al., 2011). Early diagnosis of gut dysbiosis and correction of the intestinal flora imbalance may help prevent or delay the development of HBV-ACLF. However, the relationship of the gut microbiota with disease progression, serum biomedical indicators, and fecal metabolites remains unclear.

In this study, the HBV-ACLF related gut microbiome was investigated, and alterations in the fecal microbiome over time at different stages of the progression of HBV-ACLF were determined by high-throughput $16 \mathrm{~S}$ rDNA sequencing. The relationships between HBV-ACLF specific microbiome and serum biomedical indicators and fecal metabolites were also analyzed.

\section{MATERIALS AND METHODS}

\section{Sample Collection}

A total of 91 patients with HBV-ACLF in the Department of Infection Diseases of the Second Affiliated Hospital of Nanchang University were enrolled for sampling from November 2017 to April 2018. Patients with HBV-ACLF were defined in accordance with the consensus of the Asian Pacific Association for the study of the Liver (APASL) (Sarin et al., 2014; Sarin et al., 2019): with hepatitis, rapidly develops jaundice with total bilirubin (TBIL) $>5 \mathrm{mg} / \mathrm{dl}(85 \mathrm{mmol} / \mathrm{L})$ and coagulopathy (international normalized ratio (INR) $>1.5$ or prothrombin activity (PTA) <40\%), and ascites or hepatic encephalopathy within 4 weeks. Severity grading and the therapeutic protocol of HBV-ACLF were performed as recommended by APASL. All individuals in the healthy control $(\mathrm{HC})$ group were selected by healthy physical examination results with no history of liver and gastrointestinal diseases, no previous high blood pressure, diabetes and other systemic disease, and no history of surgery within 6 months. In addition, the HC group had no HBV infection with normal liver function and renal function. Patients with HBV-ACLF who had received antibiotic treatment 3 weeks before stool or fecal sample collection were excluded. Sampling and all subsequent steps described in the Materials and Methods have been conducted in accordance with the approved guidelines. Clinical information was collected in accordance with standard procedures.

All stool and blood samples were collected after the diagnosis of HBV-ACLF stages of the patients, and the corresponding clinical data were recorded to assess the grade. A cohort of nine patients was sampled continuously at each stage of HBV-ACLF after treatment (Phase 1: rising stage (ascending stage); Phase 2: plateau stage and Phase 3: recovery stage). Note that none of the patients was at pre-rise stage, as patients at this stage are usually not yet hospitalized. The stool samples of the nine cohort patients were divided into equal portions, one for fecal flora detection and the other for metabolites analysis. After collection, the fecal samples were immediately frozen and stored at $-70^{\circ} \mathrm{C}$ before analysis. The blood samples of all patients were measured for the serum biomedical indicators: alanine transaminase (ALT); aspartate transaminase (AST); total bilirubin (TBIL); prothrombin activity (PTA); international normalized ratio (INR); WBC (white blood cells); PLT (platelet); AFP (alphafetoprotein) (Table $\mathbf{1}$ ).

\section{DNA Extraction and Sequencing}

Total genomic DNA was extracted from fecal samples using the QIAamp DNA Stool MiniKit. DNA samples were quantified using a Qubit 2.0 Fluorometer (Invitrogen, Carlsbad, CA, USA). PCR amplicons targeted with the V3 and V4 regions of $16 \mathrm{~S}$ rRNA were generated as previously described by Kozich et al. (2013), and primer 341F/806R was used to amplify the V34 region of the $16 \mathrm{~S}$ rRNA gene. Then, the PCR products were checked for size and specificity by agarose gel electrophoresis and purified. Finally, the amplicons were then sequenced using Illumina MiSeq in accordance with the manufacturer's instructions (Illumina, San Diego, CA, USA) (Klindworth et al., 2013). Sequencing was performed using a $2 \times 250$ paired-end configuration; image analysis and base calling were conducted by MiSeq Control Software embedded in the MiSeq instrument.

\section{Bioinformatic Analysis}

The raw reads were filtered to remove low-quality and polyclonal sequences in QIIME(Version 1.9.1) (Kuczynski et al., 2011). The filtered data were further compared with the Gold database and the chimera reads were detected using the Uchime algorithm in Usearch software(Version 8.1.1861) (Edgar, 2013). Resulting reads for each sample were clustered into operational 
TABLE 1 | Clinical characteristics and of the subjects in this study.

\begin{tabular}{|c|c|c|c|c|}
\hline Variables & Phase 1 & Phase 2 & Phase 3 & p-Value* \\
\hline Age (year) & $44.9 \pm 12.5$ & $41.2 \pm 13.4$ & $48.7 \pm 7.6$ & \\
\hline Male (Number) & $31(79 \%)$ & $29(88 \%)$ & $30(83 \%)$ & \\
\hline $\operatorname{ALT}(I U / I)$ & $436.10 \pm 959.54$ & $268.67 \pm 520.41$ & $62.721 \pm 61.06$ & $0.048^{\star}$ \\
\hline AST (IU/L) & $375.60 \pm 767.07$ & $146.81 \pm 224.43$ & $62.78 \pm 40.50$ & $0.016^{\star}$ \\
\hline TBIL ( $\mu \mathrm{mol} / \mathrm{L})$ & $280.98 \pm 136.44$ & $246.02 \pm 139.36$ & $149.15 \pm 125.71$ & $0^{\star}$ \\
\hline PTA (\%) & $33.42 \pm 7.53$ & $52.49 \pm 24.03$ & $81.44 \pm 43.79$ & $0.043^{\star}$ \\
\hline INR & $1.54 \pm 0.49$ & $1.37 \pm 0.41$ & $1.27 \pm 0.45$ & $0.042^{\star}$ \\
\hline $\operatorname{WBC}\left(\times 10^{9} / \mathrm{l}\right)$ & $5.59 \pm 2.33$ & $6.26 \pm 2.17$ & $5.07 \pm 1.85$ & 0.075 \\
\hline $\operatorname{PLT}\left(\times 10^{9} /\right)^{\prime}$ & $130.38 \pm 73.02$ & $138.79 \pm 80.74$ & $146.86 \pm 94.89$ & 0.696 \\
\hline Blood ammonia ( $\mu \mathrm{mol} / \mathrm{l})$ & $57 \pm 32.52$ & $65.76 \pm 24.44$ & $68.64 \pm 25.44$ & 0.496 \\
\hline $\operatorname{AFP}(\mathrm{ng} / \mathrm{ml})$ & $191.84 \pm 273.63$ & $223.69 \pm 349.45$ & $121.84 \pm 158.10$ & 0.454 \\
\hline
\end{tabular}

*The difference is statistically significant among the groups with ANOVA.

ALT, alanine transaminase; AST, aspartate transaminase; TBIL, total bilirubin; PTA, prothrombin activity; INR, international normalized ratio; WBC, white blood cell; PLT, platelet; AFP, alpha-fetoprotein.

taxonomic units (OTUs) at 97\% similarity using the Uclust algorithm in QIIME (Version 1.9.1).

The representative sequence for each OTU was selected, and the taxonomic information was annotated using QIIME (Version 1.9.1) and the Greengene database (Version 13_8). The alpha diversity indices, including the observed OTUs, Chao's richness, Simpson's evenness, and Simpson's reciprocal indexes, were calculated using QIIME (Version 1.9.1). A Principal Coordinate Analysis (PCoA) based on the weighted and unweighted UniFrac distances was conducted to compare all samples. Linear discriminant analysis (LDA) effect size (LEfSe) was measured using the Kruskal-Wallis rank sum test to detect features with significantly different abundances at the phylum and genus levels among the HC, Phase 1, Phase 2, and Phase 3. Spearman's correlation coefficients between genera and clinical indices were calculated and visualized using $\mathrm{R}$ software (Version 3.4.1).

\section{${ }^{1} \mathrm{H}$ NMR Spectroscopy of Stool Specimen}

Stool samples were prepared for ${ }^{1} \mathrm{H}$ NMR spectroscopy as previously described (Xie et al., 2014). The metabolites were identified in the ${ }^{1} \mathrm{H}$ NMR spectra by comparing their chemical shifts and coupling patterns with the corresponding values from the literature and publicly accessible databases (http://www. bmrb.wisc.edu, http://www.hmdb.ca). Total correlation spectroscopy (TOCSY), 13C-heteronuclear single-quantum correlation (13C-HSQC), statistical total correlation spectroscopy (STOCSY), and standard compounds were also utilized to identify the metabolites.

\section{Statistical Analysis}

The Shannon and Simpson indexes were calculated with QIIME (Version 1.9.1). PCoA was performed and displayed using the ade4, cluster, fpc, and cluster Sim packages in $\mathrm{R}$ software (Version 3.4.1).

Data are expressed as mean \pm standard deviation (SD) for continuous variables. ANOVA was performed to evaluate the difference followed by Tukey post hoc test. Continuous variables were compared using an independent t-test (for normally distributed ones) or nonparametric test, such as the Wilcoxon test (for those with skewed distribution) in SPSS version 11.0 for
Windows (SPSS Inc., Chicago, IL, USA). Group differences in the distribution of categorical variables were analyzed using Pearson's chi-squared test. All $\mathrm{p}$ values for bacterial microbiome analyses were corrected using the BenjaminiHochberg false discovery rate (FDR) correction, and the resulting corrected values were referred to as $\mathrm{q}$ values. The $\mathrm{q}$ values lower than 0.05 were accepted as significant. Correlation between variables was tested using Spearman rank correlation and visualized by Cytoscape (Version 3.2.1).

\section{Data Accessibility}

The raw sequence data reported in this paper have been deposited in the Genome Sequence Archive (Genomics, Proteomics \& Bioinformatics 2017) in the National Genomics Data Center (Nucleic Acids Res 2021), the China National Center for Bioinformation/Beijing Institute of Genomics, Chinese Academy of Sciences, under accession number CRA003748, which is publicly accessible at https://bigd.big.ac. cn/gsa (Wang et al., 2017; C.-N. Members, and Partners, 2021).

\section{Ethical Approval}

This study was approved by the Institutional Review Board of Second affiliated hospital of Nanchang University (KBSMC 2013-01-245-008, registered December 23, 2013). Written informed consents for all participants were obtained.

\section{RESULTS}

\section{Sample Composition and $16 S$ Amplicon Sequencing}

Among the 91 patients enrolled in our study, only nine patients had stool samples collected at all three stages of HBV-ACLF during treatment (with 27 specimens in total collected); the rest 82 patients had samples collected once per person from one of the three stages. Of the 109 stool samples, 39 were collected at the ascending stage, 34 were at the plateau stage, and 36 were at the recovery stage. Samples from $30 \mathrm{HC}$ were also collected.

16S rDNA was amplified from all 139 samples and sequenced. A total of $8,055,507$ reads were obtained and passed the quality filters. On average, each sample had 57,953 reads, which were 
assigned to 811 OTUs. Of these OTUs, $99.4 \%$ were successfully assigned at the phylum level and $55.2 \%$ at the genus level. Rarefaction curve analysis was performed to determine whether the microbiome diversity was adequately captured. For majority of the samples, the number of observed OTUs plateaued after 20,000 reads.

\section{Microbiota Changes in Patients With HBV-ACLF}

Among all HBV-ACLF samples, Firmicutes, Bacteroidetes, Proteobacteria, and Actinobacteria were the top four predominant phyla (Figure 1A). The abundance of Firmicutes, Proteobacteria, and Actinobacteria increased sharply, whereas that of Bacteroidetes decreased in the HBV-ACLF group compared with the HC group ( $\mathrm{p}<0.05$, Wilcoxon rank-sum test; Figure 1A). The intestinal flora of patients with HBV-ACLF was mainly represented by a different bacterial abundance from the $\mathrm{HC}$ group at the phylum level (seven phyla) and at the genus level (23 genera) ( $p<0.05$, Wilcoxon rank-sum test; Figures 1A, B). The overall intestinal microbiota structure was significantly different among the three stages of HBV-ACLF. The observed operational taxonomic units (OTUs) $(p=0.019)$, Simpson index $(\mathrm{p}=0.032)$, and Shannon index $(\mathrm{p}=0.0047)$, which reflect the alpha diversity, were significantly lower in the HBV-ACLF group than in the HC group ( $\mathrm{p}<0.05$ ) (Figures 2C, E). The Shannon and Simpson indexes were reduced from the early phase to the recovery phase (from phases 1 to 3 ). At the early ascending phase (phase 1), the proportion of Veillonella, Streptococcus, Enterococcus, Klebsiella, Lactobacillus, and Blautia increased, whereas the proportion of Bacteroidetes, Prevotella, and Megamonas decreased significantly $(\mathrm{p}<0.05)$ compared with those in the HC group. In the plateau phase (phase 2), the proportion of Veillonella and Enterococcus continued to rise, and the proportion of Bacteroidetes decreased again. In the recovery phase (phase 3), the proportion of Bacteroidetes and Megamonas increased, and the proportion of Enterococcus decreased. Several genera present in the HBV-ACLF groups were absent in the HC group (e.g., Lactobacillales and Streptococcus) ( $\mathrm{p}<0.05$, Wilcoxon rank-sum test; Figures 1B, 3A). At genus level, Veillonella, Streptococcus, Enterococcus, Fusobacterium, and Klebsiella were significantly more abundant in the HBV-ACLF group than in the HC group, whereas Bacteroides, Ruminococcus, Butyricimonas, Lachnospira and Sutterella were less abundant in the HBV-ACLF group than in the HC group (FDR-corrected $\mathrm{p}<0.05$, Wilcoxon rank-sum test; (Figures 1B, 3A, 4). A significant decreasing trend was observed in the Chaol index, Shannon's diversity index, and OTU index between the HBV-ACLF and HC groups. PCoA was performed using UniFrac dissimilarity data. The distance between HBV-HCLF groups showed a significant separation after the pairwise comparison of weighted UniFrac distance when compared with the distances to the control group (Figures 2A, B) with Permutation test $(p=0.001)$.

\section{LEfSe Analysis}

From the functional composition of each sample, LEfSe (LDA effect size) analysis was performed to determine the significant difference between the HBV-ACLF and HC groups (Figures 3B, C). Three predominant phyla (Firmicutes, Proteobacteria, and Actinobacteria) could be used as distinguishing biomarkers. Firmicutes, Proteobacteria, and Actinobacteria were significantly more abundant in the fecal microbiota of the HBV-ACLF group than in that of the HC group, whereas Bacteroidetes was significantly less abundant in the fecal microbiota of the HBV-ACLF group than in that of the HC group ( $\mathrm{p}<0.05$, Figure 3B).

\section{Correlation Analysis of Genus Abundance With Fecal Metabolites}

Twenty two fecal metabolites with 14 known metabolites were detected and used to determine the correlation between fecal metabolites and genus abundance. Significant correlation was found ( $p<0.05$ ) and 124 genera were associated with the metabolites (Figure 5A). The abundance of Christensenella, Clostridium, Corynebacterium, Granulicatella, and Rothia were strongly positively correlated with choline, citric acid, and pyruvic acid. The abundance of Klebsiella, Enterobacter, Bifidobacterium, Leuconostoc, Citrobacter, Cronobacter, Pediococcus, Vagococcus, Agrobacterium, Methylobacterium, Proteus, Salmonella, Morganella and Nitriliruptors showed a positive correlation with lactic acid, formic acid, and creatinine. The abundance of Erwinia, Klebsiella and Lactococcus showed a positive correlation with citric acid.

\section{Correlation Analysis of Genus Abundance With Serum Biomedical Indicators}

The levels of serum biomedical indicators ALT, AST, TBIL, INR, and PTA showed significant differences at different stages of HBV-ACLF ( $\mathrm{p}<0.05)$, The ALT, AST, TBIL, and INR levels showed a significant negative correlation with the increasing severities of HBV-ACLF. Only the PTA level positively correlated with the changes in the patient's condition (Table 1). The serum biomedical indicators were also found to be associated with the abundance of 20 genera. The TBIL levels were significantly positively correlated with the abundance of Veillonella, Fusobacterium, Campylobacter, Neisseria, Proteus, and Eikenella, but negatively with the abundance of Blautia, Coprococcus, Methanobrevibacter, and Methanosphaera. The INR levels were positively correlated with the abundance of Stenotrophomonas and Pseudochrobactrum, but negatively with that of Blautia, Dorea, Coprococcus, Anaerostipes, Adlercreutzia, and Methanobrevibacter. The ATL and AST levels were positively correlated with the abundance of Moryella and Fusobacterium respectively. The PTA levels were positively correlated with the abundance of Anaerostipes, Coprococcus, Adlercreutzia, Blautia and Dorea, but negatively with that of Shinella, Stenotrophomonas, and Pseudochrobactrum (Figures 5B, C).

\section{DISCUSSION}

The human microbiome can influence genetic diversity, immunity, and metabolism (Zhu et al., 2010; Grice and Segre, 2012). 
A

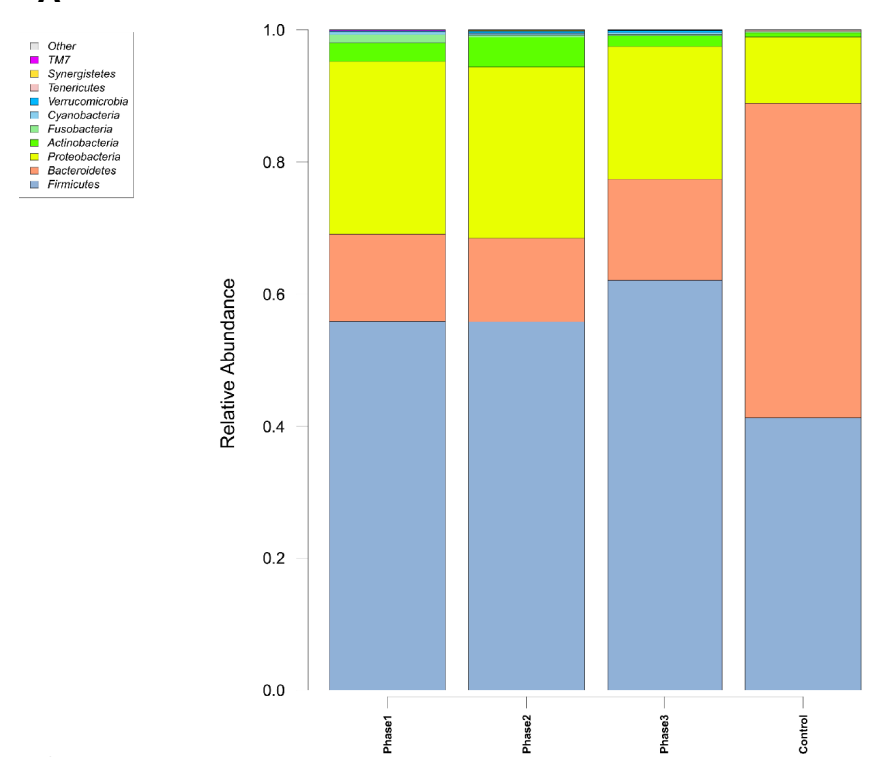

B

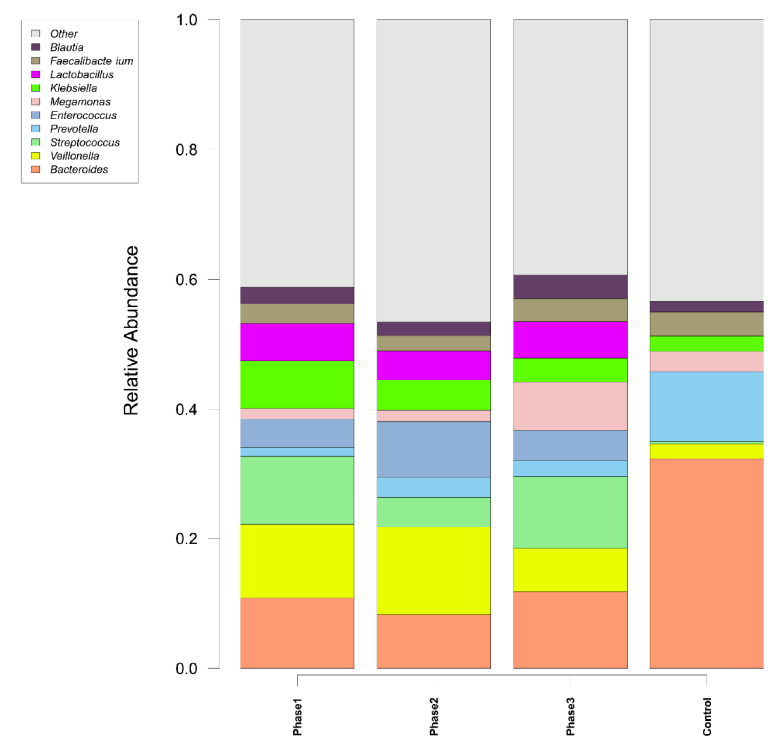

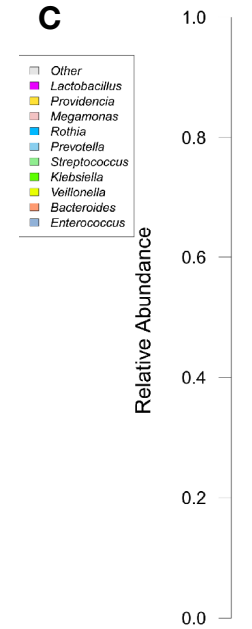

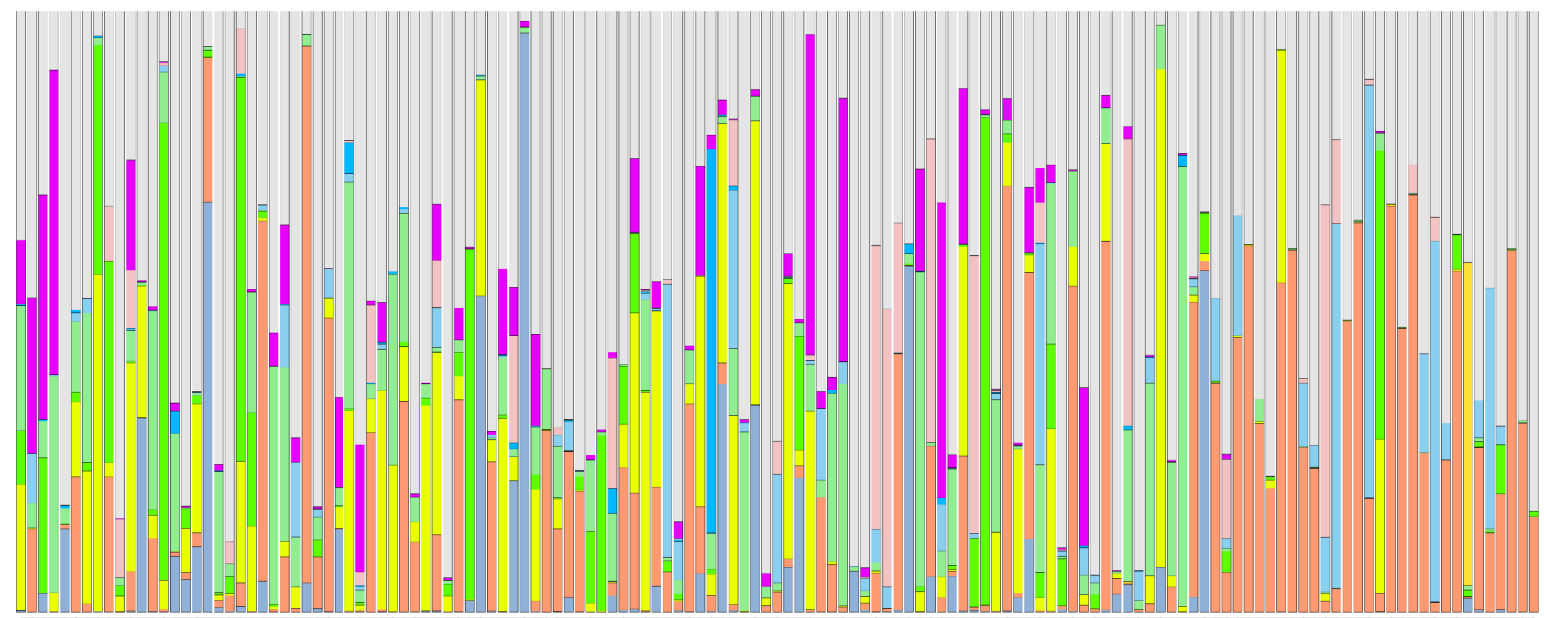

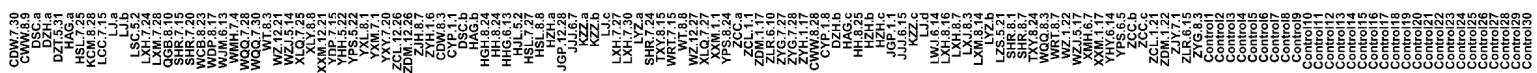

FIGURE 1 | Characteristics of gut microbiota composition. (A) Relative abundance of microbial communities at the phylum level. Phase 1: rising stage; Phase 2: plateau stage; and Phase 3: recovery stage. (B) Relative abundance of microbial communities at the genus level. Phase 1: rising stage; phase 2: plateau stage; and phase 3: recovery stage (C) Relative abundance of all 109 samples at the top 10 genus level. (Individuals are shown along the horizontal axis, and relative taxa proportions are represented by the vertical axis. The X-axis showed the each sample, the naming principle of samples were the initials of the patients' name added with the sampling date). 
A

$$
\begin{aligned}
& \text { Group } \\
& \text { - Control } \\
& \text { Phase1 } \\
& - \text { Phase2 } \\
& \hline
\end{aligned}
$$

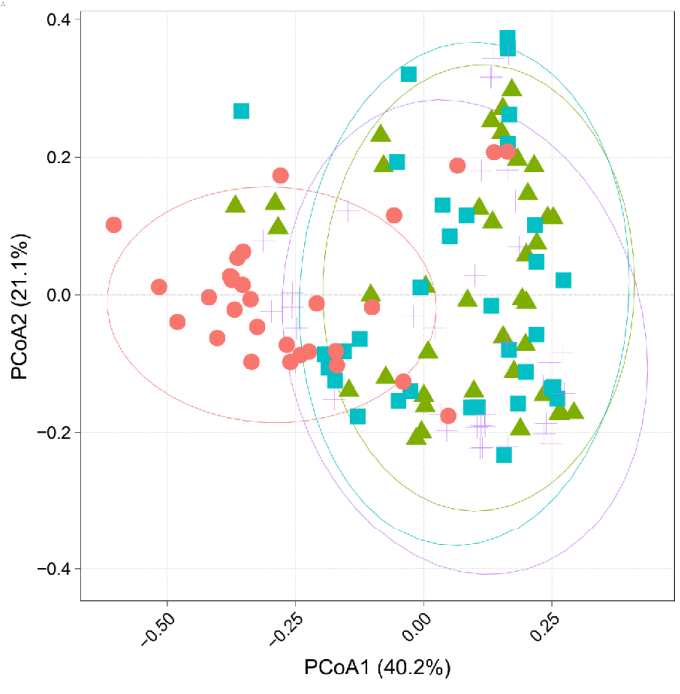

C

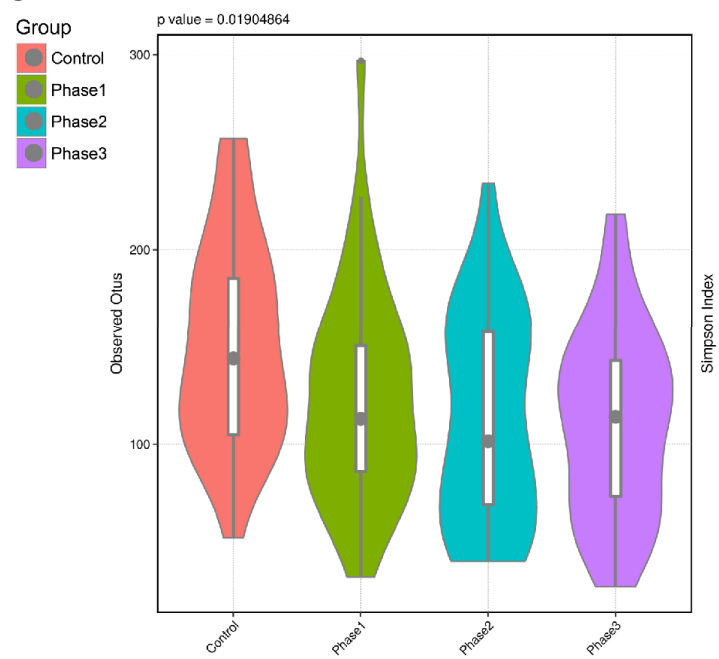

B

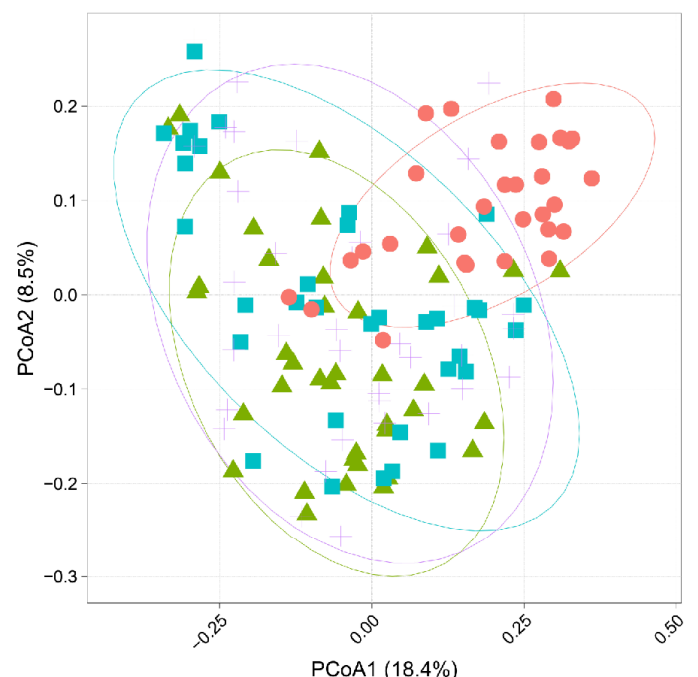

E
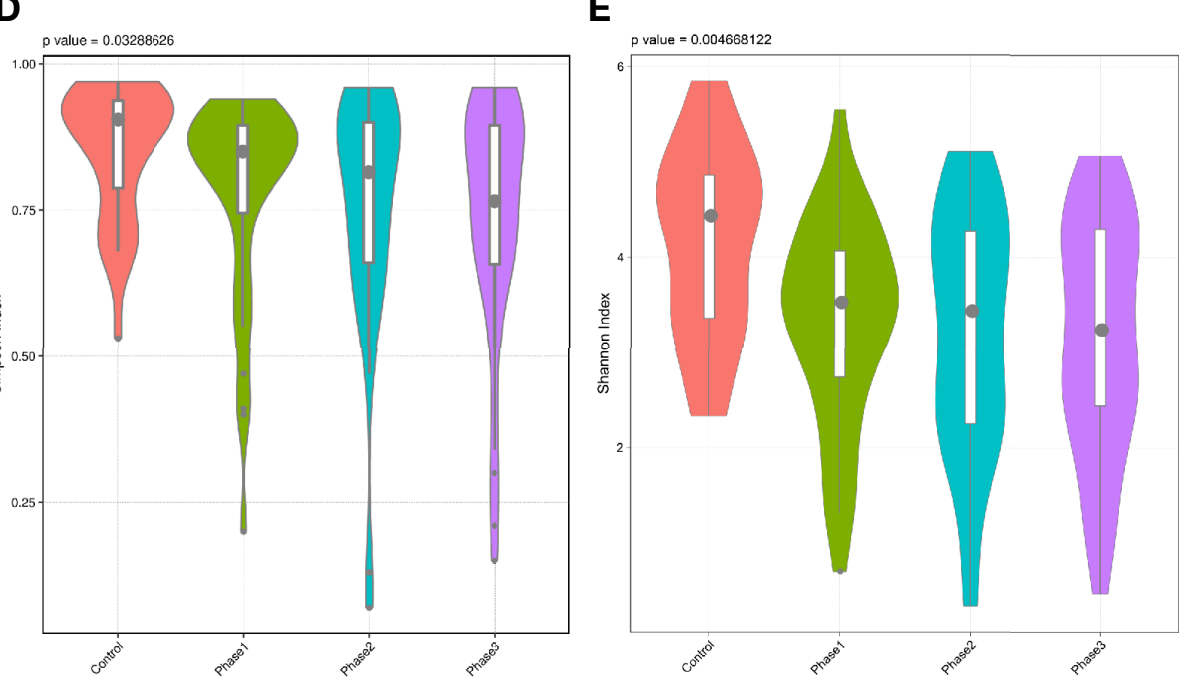

FIGURE 2 | Diversity analysis of the gut microbiota of the HBV-ACLF patients. (A) PCOA plot based on weighted unifrac distance of the three phases of HBV-ACLF and the HC group (permutation test, $p=0.001$ ). (B) PCoA plot based on unweighted unifrac distance of the three phases of HBV-ACLF and the HC group (permutation test, $\mathrm{p}=0.001$ ). (C) Operational taxonomic units (OTUs) of the three phases of HBV-ACLF and the HC group. $P=0.019$ from $t$ test. (D) Simpson index of the three phases of HBV-ACLF and the HC group ( $p=0.033$ from $t$ test). (E) Shannon index indicated the community diversity of the three phases of HBV-ACLF and HC group ( $p=0.0046$ from $t$ test). 

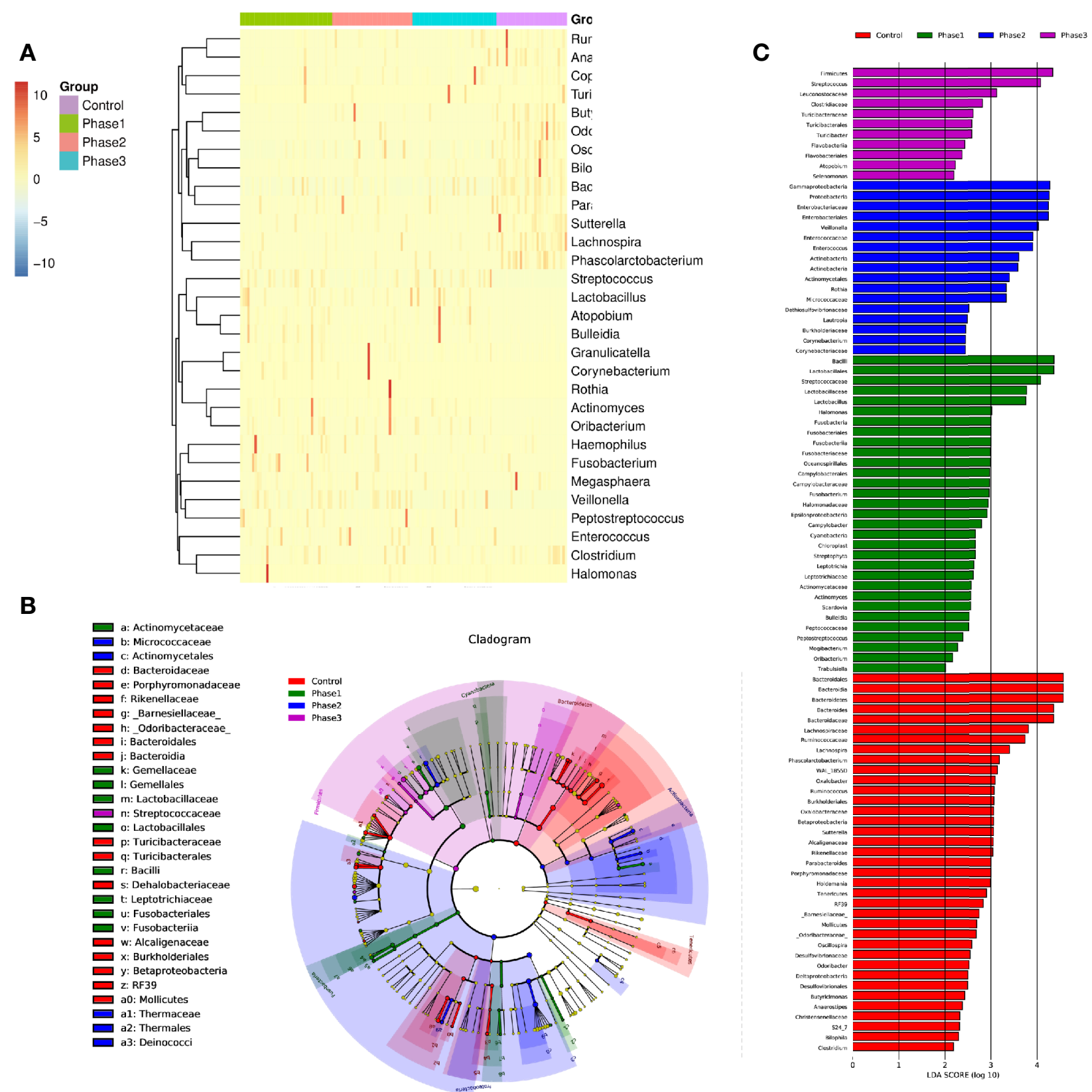

B
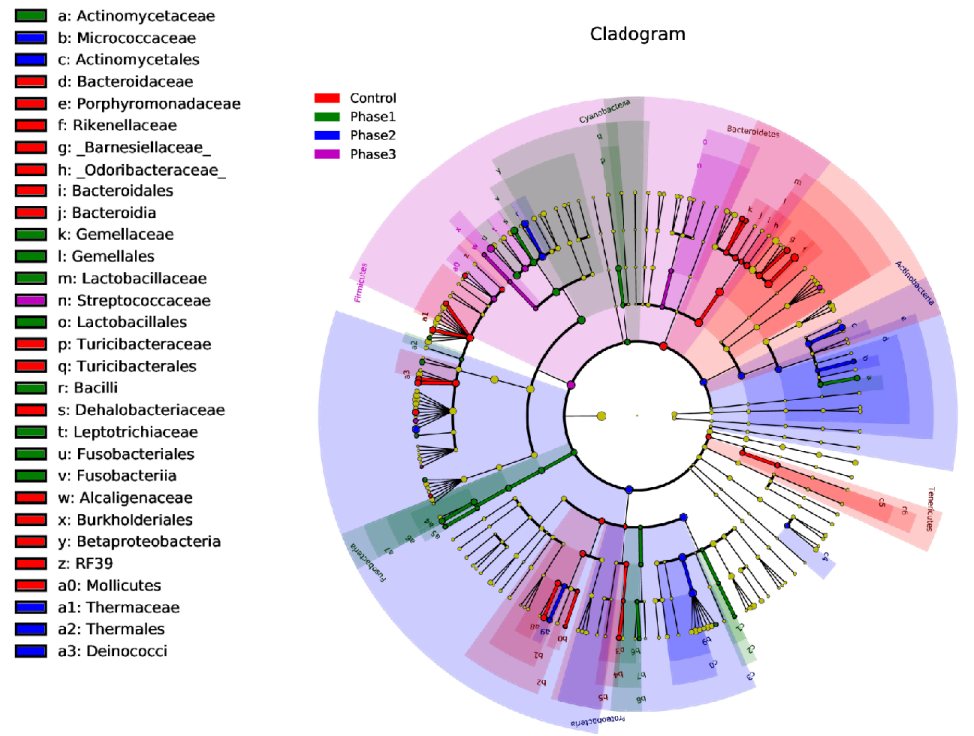

FIGURE 3 | The statistically significant microbial communities associated with the HBV-ACLF. (A) Heatmap of 32 statistically significant genera. (B) Cladogram indicated the phylogenetic distribution of microbial communities associated with the three phases and the HC group; lineages with LDA values of 3.0 or higher as determined by LEfSE are shown. LEfSe (LDA Effect Size) analysis was performed to find biomarkers with significant differences ( $p<0.05)$ between different groups. Differences are represented by the color of the most abundant class. Green sample indicates Phase 1, blue sample indicates Phase 2 , purple sample indicates Phase 3, and red represents HC group. (C) Indicator microbial groups in the four groups with LDA scores higher than 3.0. The bar length is proportional to the LD score that indicates the significance of the genus. Differences are represented by the color of the most abundant class. Green sample indicates Phase 1, blue sample indicates Phase 2, purple sample indicates Phase 3, and red represents $\mathrm{HC}$ group.

The gut microbiome is a key determinant of intestinal inflammation and plays a significant role in host metabolic processes and host immune modulation (Hawkins et al., 2020). A strong relationship exists between the liver and the gut via the portal system (the venous system of the portal circulation) (Minemura and Shimizu, 2015). The gut-liver axis highlights the close anatomical and functional interaction of the gastrointestinal tract and the live (Kang and Cai, 2017). 
A

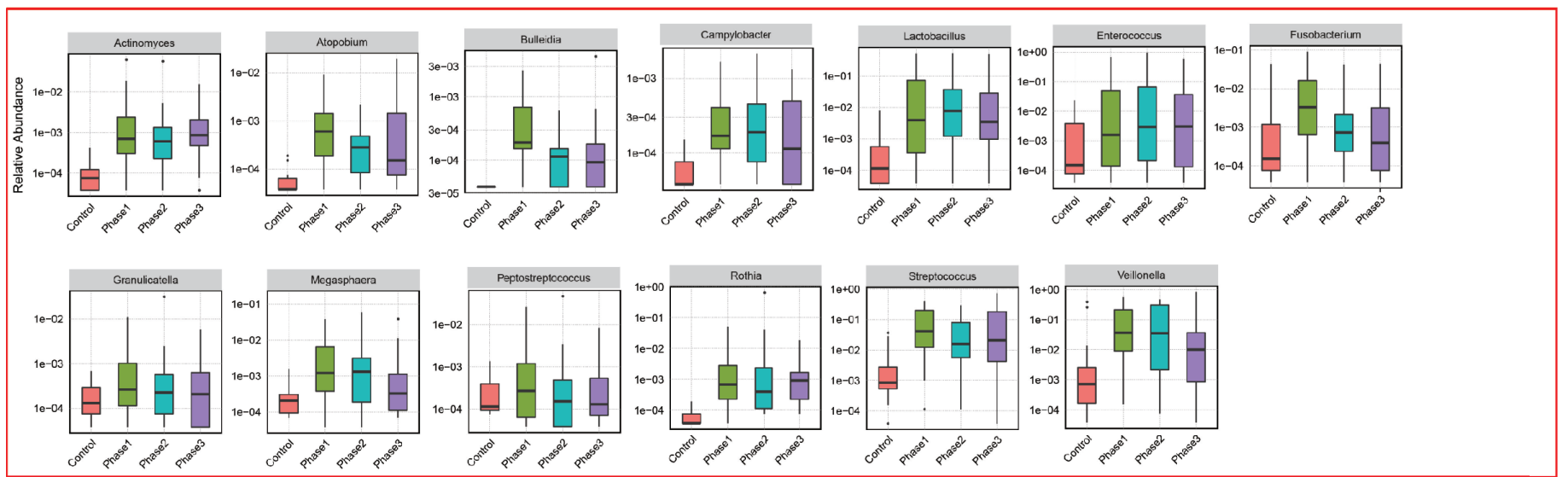

B

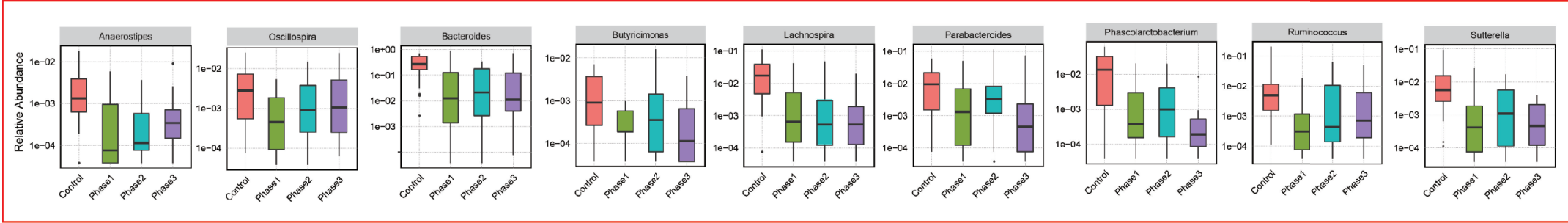

C

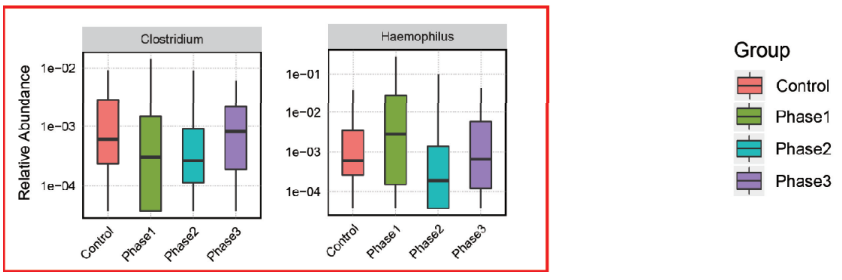

FIGURE 4 | Relative abundance of genus that was statistically significant correlated with HBV-ACLF. Phase 1 (green boxplot), phase 2 (blue boxplot), phase 3 (purple boxplot), and control (red boxplot). (A) Relative abundance was significantly increased. (B) Relative abundance was significantly reduced. (C) Uncertain. 

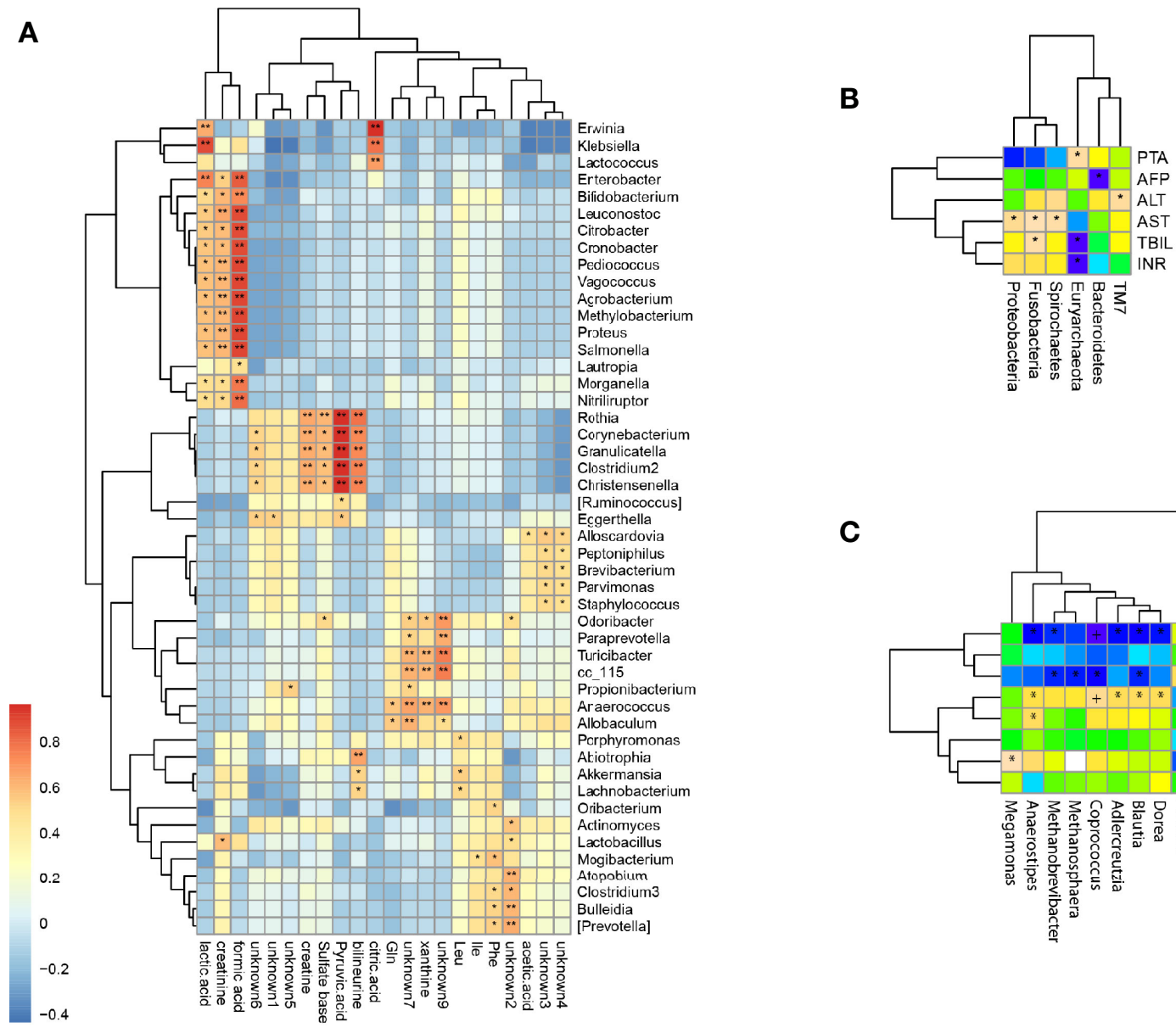

C

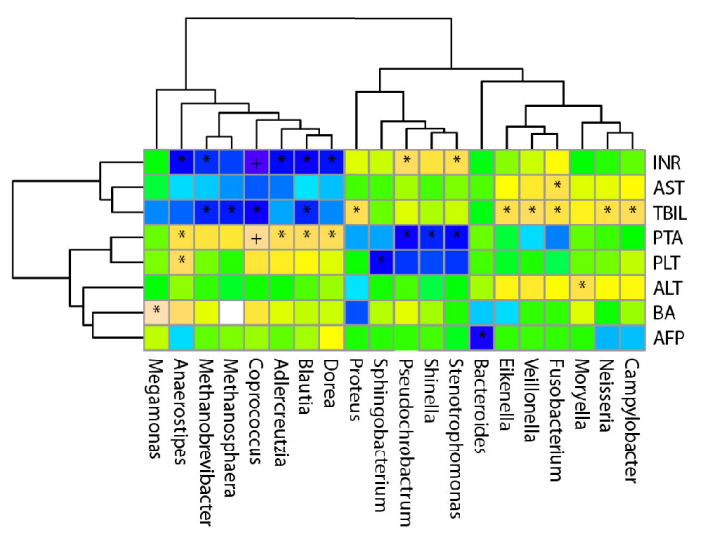

FIGURE 5 | Correlations of bacterial taxon abundance with fecal metabolites and biomedical indicators. (A) Correlations of genus abundance with fecal metabolites (asterisk means $p<0.05)$. (B) Correlation analysis of phylum abundance with patient's serum biomedical indicators (asterisk means $p<0.05)$. (C) Correlation analysis of genus abundance with patient's serum biomedical indicators (asterisk means $p<0.05$ ).

Specific microbiome patterns in stool were previously found to be present in patients with ACLF (Bajaj et al., 2014b; Zhang et al., 2019). A previous study also suggested that Lachnospiraceae was a biomarker of ACLF, whereas the relative abundance of Pasteurellaceae predicted mortality (Chen Y et al., 2015). Microbial metabolites have also been found to be associated with ACLF development (Moreau et al., 2020). In this study, diversity and abundance of the gut microbiome was found to be significantly decreased with the progression of HBV-ACLF. Bacterial taxa (phyla and genera) that were correlated positively or negatively with $\mathrm{HBV}$-ACLF were identified. Further bacterial taxa that were correlated with serum biomarkers and fecal metabolites were also identified. Thus, the dysbiosis of gut microbiome detected in HBV-ACLF patients may directly contribute to the alterations of host metabolism and progression of HBV-ACLF.

As found in our study, when the balance of intestinal flora was altered, the potential pathogens of Streptococcus, Veillonella, Klebsiella, and Enterococcus were more abundant in the HBV-
ACLF group than in the HC group, and the relative abundance of the beneficial bacteria Bacteroides was decreased. The increased abundance of pathogenic bacteria, such as Enterococcus and Klebsiella, may lead to damage the microvilli membrane of intestinal epithelial cells but also interfere with the biochemical reaction of intestinal epithelial cells. Due to dysbiosis, impairment of the intestinal micro-ecological barrier, and altered immunity status, bacterial products (such as toxins or metabolites) can reach the liver through the portal vein, where they are recognized by specific receptors, activate the immune system, and lead to a proinflammatory response (Fukui, 2015; Arab et al., 2018). Lactobacillus can stimulate intestinal dendritic cells to initiate immune response, reduce endotoxin, improve intestinal mucosal barrier function, and reduce hepatocyte injury. The HBV-ACLF patients enrolled in our study were all treated with Lactobacillus capsules to regulate the balance of intestinal microflora. Hence, the Lactobacillus was relatively more abundant in the HBV-ACLF group than in the $\mathrm{HC}$ group. Hierarchical clustering and PCoA of beta diversity discriminated the HBV-ACLF group from the $\mathrm{HC}$ 
group. The relative abundance at the phylum and genus levels was different with the development of HBV-ACLF from Phase 1 to Phase 3, with an increase in Veillonella, Klebsiella and Enterococcus in Phase 1 and a decrease in Phase 3; the abundance of Bacteroides and Megamonas decreased at Phase 1 and then increased in Phase 3. Streptococcus was abundant and enriched only in the HBV-ACLF group. Thus, the alterations of these bacterial taxa may be considered as specific biomarkers for HBV-ACLF development. A previous study showed that liver transplantation changed the gut microbiome towards increased microbial diversity, increased autochthonous bacteria (for example, Lachnospiraceae) and decreased potentially pathogenic bacteria (for example, Enterobacteriaceae) (Bajaj et al., 2017). The findings in that study were consistent with our results that the diseased liver was associated with an altered gut microbiome.

Investigation of the relationship between the gut microbiome and fecal metabolome can offer insights into the interaction of the microbiome, liver, and gut. Conversely, alteration of bacterial abundance may impact on the host and/or gut metabolism. Liver dysfunction can lead to the abnormal synthesis of important biologic signal molecules, metabolic disturbances, and alterations in serum biomedical indicators. Citric acid, pyruvic acid, lactic acid, and formic acid are the intermediate products of the tricarboxylic acid cycle, which is the common pathway of the metabolism of carbohydrates, lipids, and amino acids (Liu et al., 2017). Our results showed that the levels of citric acid, pyruvic acid, lactic acid, and formic acid in feces were strongly correlated with the abundance of 20 bacterial genera, such as Christensenella, Clostridium, Corynebacterium, Granulicatella, RothiaKlebsiella, Enterobacter, Bifidobacterium, Leuconostoc, Citrobacter, Cronobacter, Pediococcus, Vagococcus, Agrobacterium, Methylobacterium, Proteus, Salmonella, Morganella, Nitriliruptor, Erwinia, Klebsiella, and Lactococcus. These results suggest that HBV-ACLF could result in metabolic disorders and intestinal bacteriosis, thereby affecting physiological functions of the gust in the host.

In addition, significantly elevated levels of serum ALT, TBIL, and PTA often suggest serious liver damage and are often used to diagnose HBV-ACLF (Moreau et al., 2013; Sarin et al., 2014). HBV replication in hepatocytes could lead to a host immune reaction characterized by elevated ALT levels and may promote changes in bile acid and lipid metabolism in the liver and gut. We found that the HBV-ACLF patients' serum biomarkers among the different stages of HBV-ACLF showed a statistical difference in the levels of ALT, AST, TBIL, INR, and PTA ( $\mathrm{p}<0.05$ ). We also found that the abundance of different genera was altered in HBV-ACLF patients and different biomarkers were correlated with the alterations of different bacterial genera. Fifteen genera were positively correlated with one of the five biomarkers (Veillonella, Fusobacterium, Campylobacter, Neisseria, Proteus, and Eikenella with TBIL; Stenotrophomonas and Pseudochrobactrum with INR; Moryella with ATL; and Fusobacterium with AST; Anaerostipes, Coprococcus, Adlercreutzia, Blautia, and Dorea with PTA), while 10 genera were negatively correlated with one or more of the five biomarkers (Blautia, Coprococcus, Methanobrevibacter, and Methanosphaera with TBIL; Blautia, Dorea, Coprococcus, Anaerostipes, Adlercreutzia, and Methanobrevibacter with INR; Shinella, Stenotrophomonas, and Pseudochrobactrum with PTA). However some genera have opposing correlation with different biomarkers, for example, Pseudochrobactrum was positively correlated with INR level but negatively with PTA level. Our results showed high correlations of genus abundance with serum biomarkers, such as serum ALT, AST, TBIL, and INR, in patients with HBV-ACLF. Thus, gut bacterial abnormalities may play a role in the pathogenesis and development of HBV-ACLF.

\section{CONCLUSION}

High-throughput sequencing of 16S rDNA and fecal metabolite analysis were employed to investigate the impact of the gut microbiome and metabolic alterations of HBV-ACLF patients. Results showed that the diversity and abundance of the gut microbiome were perturbed in HBV-ACLF patients. Strong correlations were observed between the gut bacteria with fecal metabolites and serum biomarkers, such as serum ALT, AST, TBIL, and INR. Our findings suggest that the gut microbiota plays an important role in the pathogenesis and development of HBV-ACLF.

\section{DATA AVAILABILITY STATEMENT}

The datasets presented in this study can be found in online repositories. The names of the repository/repositories and accession number(s) can be found below: Genome Sequence Archive under accession number CRA003748.

\section{ETHICS STATEMENT}

The studies involving human participants were reviewed and approved by the Institutional Review Board of Second affiliated hospital of Nanchang University (KBSMC 2013-01-245-008, registered December 23, 2013). The patients/participants provided their written informed consent to participate in this study.

\section{AUTHOR CONTRIBUTIONS}

All authors contributed to the article and approved the submitted version. XY, JL, HY, SS, and BX prepared manuscript, writing, correction, and figures design. BX, GF, HHX, LL, HLX, ZW, MC, $\mathrm{WX}$, and ZG generated experimental data and wrote the manuscript. CL, WG, AY, KS, and RY conceived the work and critically reviewed the manuscript.

\section{FUNDING}

This work was supported by Jiangxi Provincial key research and development project No: 20171BBG70086; and National 
Natural Science Foundation of China (General Project No 81860113).

\section{ACKNOWLEDGMENTS}

We thank Professor Ruiting Lan for critical reading and helpful comments on our manuscript.

\section{REFERENCES}

Arab, J. P., Martin-Mateos, R. M., and Shah, V. H. (2018). Gut-liver axis, cirrhosis and portal hypertension: the chicken and the egg. Hepatol. Int. 12, 24-33. doi: 10.1007/s12072-017-9798-x

Bajaj, J. S., O'Leary, J. G., Reddy, K. R., Wong, F., Biggins, S. W., Patton, H., et al. (2014a). Survival in infection-related acute-on-chronic liver failure is defined by extrahepatic organ failures. Hepatology 60, 250-256. doi: 10.1002/hep.27077

Bajaj, J. S., Heuman, D. M., Hylemon, P. B., Sanyal, A. J., White, M. B., Monteith, P., et al. (2014b). Altered profile of human gut microbiome is associated with cirrhosis and its complications. J. Hepatol. 60, 940-947. doi: 10.1016/ j.jhep.2013.12.019

Bajaj, J. S., Fagan, A., Sikaroodi, M., White, M. B., Sterling, R. K., Gilles, H., et al. (2017). Liver transplant modulates gut microbial dysbiosis and cognitive function in cirrhosis. Liver Transplantation 23, 907-914. doi: 10.1002/lt.24754

C.-N. Members, and Partners (2021). Database Resources of the National Genomics Data Center, China National Center for Bioinformation in 2021. Nucleic Acids Res. 49, D18-D28. doi: 10.1093/nar/gkaa1022

Chen, E. Q., Zeng, F., Zhou, L. Y., and Tang, H. (2015). Early warning and clinical outcome prediction of acute-on-chronic hepatitis B liver failure. World J. Gastroenterol. 21, 11964-11973. doi: 10.3748/wjg.v21.i42.11964

Chen, D., Wen, S., Wu, Z., Gu, Y., Chen, Y., Chen, X., et al. (2021). Association between effective hepatic blood flow and the severity and prognosis of hepatitis B virus-related acute on chronic liver failure. Eur. J. Gastroenterol. Hepatol. 32, 246-254. doi: 10.1097/MEG.0000000000001721

Chen, Y., Guo, J., Qian, G., Fang, D., Shi, D., Guo, L., et al. (2015). Gut dysbiosis in acute-on-chronic liver failure and its predictive value for mortality. J. Gastroenterol. Hepatol. 30, 1429-1437. doi: 10.1111/jgh.12932

Edgar, R. C. (2013). UPARSE: highly accurate OTU sequences from microbial amplicon reads. Nat. Methods 10, 996-998. doi: 10.1038/nmeth.2604

Fukui, H. (2015). Gut Microbiota and Host Reaction in Liver Diseases. Microorganisms 3, 759-791. doi: 10.3390/microorganisms3040759

Grice, E. A., and Segre, J. A. (2012). The human microbiome: our second genome. Annu. Rev. Genomics Hum. Genet. 13, 151-170. doi: 10.1146/annurev-genom090711-163814

Hawkins, K. G., Casolaro, C., Brown, J. A., Edwards, D. A., and Wikswo, J. P. (2020). The Microbiome and the Gut-Liver-Brain Axis for Central Nervous System Clinical Pharmacology: Challenges in Specifying and Integrating In Vitro and In Silico Models. Clin. Pharmacol. Ther. 108 (5), 929-948. doi: $10.1002 /$ cpt. 1870

Kang, Y., and Cai, Y. (2017). Gut microbiota and hepatitis-B-virus-induced chronic liver disease: implications for faecal microbiota transplantation therapy. J. Hosp. Infection 96, 342-348. doi: 10.1016/j.jhin.2017.04.007

Klindworth, A., Pruesse, E., Schweer, T., Peplies, J., Quast, C., Horn, M., et al. (2013). Evaluation of general $16 \mathrm{~S}$ ribosomal RNA gene PCR primers for classical and next-generation sequencing-based diversity studies. Nucleic Acids Res. 41, el. doi: 10.1093/nar/gks808

Kozich, J. J., Westcott, S. L., Baxter, N. T., Highlander, S. K., and Schloss, P. D. (2013). Development of a dual-index sequencing strategy and curation pipeline for analyzing amplicon sequence data on the MiSeq Illumina sequencing platform. Appl. Environ. Microbiol. 79, 5112-5120. doi: 10.1128/AEM.01043-13

Kuczynski, J., Stombaugh, J., Walters, W. A., Gonzalez, A., Caporaso, J. G., and Knight, R. (2011). Using QIIME to analyze $16 \mathrm{~S}$ rRNA gene sequences from microbial communities. Curr. Protoc. Bioinf. Chapter 10Unit 10, 7. doi: 10.1002/0471250953.bi1007s36

\section{SUPPLEMENTARY MATERIAL}

The Supplementary Material for this article can be found online at: https://www.frontiersin.org/articles/10.3389/fcimb.2021. 573923/full\#supplementary-material

Supplementary Table 1 | Relative abundance of all 109 samples at the top 10 genus level.

Liu, D., Li, T., Zheng, H., Yin, X., Chen, M., Liao, Z., et al. (2017). Study on alterations of physiological functions in aged constipation rats with fluiddeficiency based on metabonomic and microbiology analysis. R. Soc. Chem. Adv. 7, 48136-48150. doi: 10.1039/C7RA07651G

Minemura, M., and Shimizu, Y. (2015). Gut microbiota and liver diseases. World J. Gastroenterol. 21, 1691-1702. doi: 10.3748/wjg.v21.i6.1691

Moreau, R., Jalan, R., Gines, P., Pavesi, M., Angeli, P., Cordoba, J., et al. (2013). Acute-on-chronic liver failure is a distinct syndrome that develops in patients with acute decompensation of cirrhosis. Gastroenterology 144, 1426-1437. doi: 10.1053/j.gastro.2013.02.042

Moreau, R., Clària, J., Aguilar, F., Fenaille, F., Lozano, J. J., Junot, C., et al. (2020). Blood metabolomics uncovers inflammation-associated mitochondrial dysfunction as a potential mechanism underlying ACLF. J. Hepatol. 72, 688701. doi: 10.1016/j.jhep.2019.11.009

Ott, J. J., Horn, J., Krause, G., and Mikolajczyk, R. T. (2017). Time trends of chronic HBV infection over prior decades - A global analysis. J. Hepatol. 66, 48-54. doi: 10.1016/j.jhep.2016.08.013

Preveden, T., Scarpellini, E., Milic, N., Luzza, F., and Abenavoli, L. (2017). Gut microbiota changes and chronic hepatitis $\mathrm{C}$ virus infection. Expert Rev. Gastroenterol. Hepatol. 11, 813-819. doi: 10.1080/17474124.2017.1343663

Sandler, N. G., Koh, C., Roque, A., Eccleston, J. L., Siegel, R. B., Demino, M., et al. (2011). Host response to translocated microbial products predicts outcomes of patients with HBV or HCV infection. Gastroenterology 141, 1220-1230. doi: 10.1053/j.gastro.2011.06.063

Sarin, S. K., Kedarisetty, C. K., Abbas, Z., Amarapurkar, D., Bihari, C., Chan, A. C., et al. (2014). Acute-on-chronic liver failure: consensus recommendations of the Asian Pacific Association for the Study of the Liver (APASL) 2014. Hepatol. Int. 8, 453-471. doi: 10.1007/s12072-014-9580-2

Sarin, S. K., Choudhury, A., Sharma, M. K., Maiwall, R., Al Mahtab, M., Rahman, S., et al. (2019). Acute-on-chronic liver failure: consensus recommendations of the Asian Pacific association for the study of the liver (APASL): an update. Hepatol. Int. 13, 353-390. doi: 10.1007/s12072-019-09946-3

Tilg, H., Cani, P. D., and Mayer, E. A. (2016). Gut microbiome and liver diseases. Gut 65, 2035-2044. doi: 10.1136/gutjnl-2016-312729

Tsai, W. L., Sun, W. C., and Cheng, J. S. (2015). Chronic Hepatitis B with Spontaneous Severe Acute Exacerbation. Int. J. Mol. Sci. 16, 28126-28145. doi: 10.3390/ijms 161226087

Uesugi, T., Froh, M., Arteel, G. E., Bradford, B. U., Wheeler, M. D., Gäbele, E., et al. (2002). Role of lipopolysaccharide-binding protein in early alcohol-induced liver injury in mice. J. Immunol. 168, 2963-2969. doi: 10.4049/ jimmunol.168.6.2963

Verbeke, L., Nevens, F., and Laleman, W. (2011). Bench-to-beside review: acuteon-chronic liver failure - linking the gut, liver and systemic circulation. Crit. Care 15, 233. doi: 10.1186/cc10424

Wang, B., and Li, L. (2015). Who determines the outcomes of HBV exposure? Trends Microbiol. 23, 328-329. doi: 10.1016/j.tim.2015.04.001

Wang, Y., Song, F., Zhu, J., Zhang, S., Yang, Y., Chen, T., et al. (2017). GSA: Genome Sequence Archive. Genomics Proteomics Bioinf. 15, 14-18. doi: 10.1016/j.gpb.2017.01.001

Wlodzimirow, K. A., Eslami, S., Abu-Hanna, A., Nieuwoudt, M., and Chamuleau, R. A. (2012). Systematic review: acute liver failure - one disease, more than 40 definitions. Alimentary Pharmacol. Ther. 35, 1245-1256. doi: 10.1111/j.13652036.2012.05097.x

Xie, B., Liu, A., Zhan, X., Ye, X., and Wei, J. (2014). Alteration of gut bacteria and metabolomes after glucaro-1,4-lactone treatment contributes to the prevention of hypercholesterolemia. J. Agric. Food Chem. 62, 7444-7451. doi: 10.1021/jf501744d 
Xing, T., Li, L., Cao, H., and Huang, J. (2007). Altered immune function of monocytes in different stages of patients with acute on chronic liver failure. Clin. Exp. Immunol. 147, 184-188.

Ye, Y., and Gao, Z. (2009). Three shock hypotheses that may induce liver failure. Chin. J. Hepatol. 8, 638-640. doi: 10.1111/j.1365-2249.2006.03259.x

Zang, G., Zhou, X., Yu, H., Xie, Q., Zhao, G., Wang, B., et al. (2000). Effect of hepatocyte apoptosis induced by TNF-alpha on acute severe hepatitis in mouse models. World J. Gastroenterol 6, 688-692. doi: 10.3748/wjg.v6.i5.688

Zhang, T., Sun, K., Wang, Y., Huang, L., Lang, R., and Jiang, W. (2018). Disruption of the gut-liver axis in the pathogenesis of acute-on-chronic liver failure. Eur. J. Gastroenterol. Hepatol. 30, 130-135. doi: 10.1097/MEG.0000000000001026

Zhang, Y., Zhao, R., Shi, D., Sun, S., Ren, H., Zhao, H., et al. (2019). Characterization of the circulating microbiome in acute-on-chronic liver failure associated with hepatitis B. Liver Int. 39, 1207-1216. doi: 10.1111/liv.14097
Zhu, B., Wang, X., and Li, L. (2010). Human gut microbiome: the second genome of human body. Protein Cell 1, 718-725. doi: 10.1007/s13238-010-0093-z

Conflict of Interest: The authors declare that the research was conducted in the absence of any commercial or financial relationships that could be construed as a potential conflict of interest.

Copyright (C) 2021 Yao, Yu, Fan, Xiang, Long, Xu, Wu, Chen, Xi, Gao, Liu, Gong, Yang, Sun, Yu, Liang, Xie and Sun. This is an open-access article distributed under the terms of the Creative Commons Attribution License (CC BY). The use, distribution or reproduction in other forums is permitted, provided the original author(s) and the copyright owner(s) are credited and that the original publication in this journal is cited, in accordance with accepted academic practice. No use, distribution or reproduction is permitted which does not comply with these terms. 